\title{
From Ibni Sina (Avicenna) to Present, History of International Fellowship and Observership: University of Wisconsin-Madison Experience
}

\author{
Sima SAYYAHMELLI, Yannick Canton KESSELY, Xinpu CHEN, Abdurrahman AYCAN, Santiago FELDMAN, \\ Robert J. DEMPSEY, Mustafa K. BASKAYA
}

University of Wisconsin-Madison, School of Medicine and Public Health, Department of Neurological Surgery, Madison, WI, USA

Corresponding author: Mustafa K. BASKAYA baskaya@neurosurgery.wisc.edu

\section{ABSTRACT}

Since the inception of the International Fellowship (IF) Program in the Department of Neurological Surgery at the University of Wisconsin-Madison in 2006, training has been provided to 219 residents, neurosurgeons, and medical students from 18 countries and five continents. These IFs took a long academic and geographic journey to improve their skills in patient care. The advanced training, they received lead to 14 of these IF neurosurgeons to return to their hometowns with higher academic appointments, including two chairmen, seven professors, two associate professors, two assistant professors, and one consultant neurosurgeon. An additional measure of success for the IF Program is that fellows continue to communicate with their mentors and with their prior fellow international colleagues long after their fellowship ends.

KEYWORDS: Education, International Fellowship Program, Observership, Neurosurgery, Training, University of Wisconsin-Medical School

\section{INTRODUCTION}

$\mathrm{T}$ The International Fellowship (IF) Program in the Department of Neurological Surgery at the University of Wisconsin-Madison provides advanced training in neuroscience and neurosurgery for international neurosurgeons, neurosurgery residents, medical students, and undergraduate students. Since its inception in 2006, training has been provided to 219 residents, neurosurgeons, and medical students from 18 countries. Upon completion of their fellowship, these physician-scientists have then returned to advance the quality of therapeutic care in their home county.

The University of Wisconsin Department of Neurological Surgery provides world-class education and research opportunities to fellows. Fellows receive research scholar appointments that qualify for the $\mathrm{J} 1$-Exchange program in the United States of America (USA).
In the following, we describe our experience as hosts of this program over its 14-year history, and provide an overview of the curriculum and the experiences of its international trainees.

\section{History of Observership in Medicine}

Ibni Sina, also known as Abu Ali Sina and as Avicenna, revolutionized medicine. Avicenna died at the age of 57 in 1037 in Persia (2). In his famous five-volume book, "The Canon of Medicine", (2) he described his observations of diverse illnesses and his perceptions of rare disorders. Over his lifetime, he educated hundreds of trainees who traveled from diverse geographical regions. Avicenna's principal teaching method was to "observe." This tenet of "observation" has continued to the present in the medical schools of his home country, where they consider medicine an experimental major. Trainees are instructed to closely observe the masters and pioneers of each proficiency in order to accustom themselves
Sima SAYYAHMELLI

(D) : 0000-0001-8745-6497

Yannick Canton KESSELY (1) : 0000-0003-1092-1017

Xinpu CHEN
(D) : 0000-0002-6895-8296
Abdurrahman AYCAN (1) : 0000-0002-3794-8511

Santiago FELDMAN (D): 0000-0003-4234-3397

Robert J. DEMPSEY (1) : 0000-0002-1636-5456
Mustafa K. BASKAYA (D) : 0000-0002-2782-4793 
to each specific field and methodology. This tradition of "observation" foremost continues today in major teaching centers throughout the developed world. International trainees come to different hospitals in the developed countries to observe advances in knowledge and technologies, and to improve their career by observing the masters and pioneers in their intended field, including in neurological surgery. As eloquently stated by Professor Peter Jannetta, MD (Chairman of the Department of Neurosurgery, University of Pittsburgh from 1971-96), "One surgeon, no matter how much he or she operates, has very little effect. But if he or she trains a group of surgeons who go forth and multiply and train others, then the ripple effect is tremendous" (1).

\section{Importance of Global Neurosurgical Training}

A dedicated global neurosurgical association can expand the current panorama by educating neurosurgeons, and by expediting the transmission of fundamental neurosurgical knowledge and instrumentation to all needed regions worldwide (11). Creating a curriculum for global education is now being achieved through the joint endeavors of the Society of Neurological Surgeons and the Foundation for International Education in Neurological Surgery, also known as FIENS (36,11 ). By including doctors from each geographic region in the didactic curriculum, each region thus can independently provide the appropriate training to meet the needs of its citizens (6). Greater than one-third of the worldwide population live in low-income countries, yet this population receives only about $6 \%$ of surgical procedures each year (9). Mukhopadhyay et al. recently estimated that there are 49,940 neurosurgeons worldwide, with the vast majority in developed countries, and that there are fewer than 5 neurosurgeons per million population in most of the countries of Africa, the Middle East and the Indian subcontinent (10).

Clearly, improving access to fundamental neurosurgical care in low- and middle-income countries is crucial to decrease the burden of neurological disease (7). Despite worldwide efforts in this regard, we are still only beginning to improve global neurosurgical care (8). In this report, we describe how our 14-year experience of hosting international fellows at the University of Wisconsin has provided significant beneficial effects to promote global neurosurgical care.

\section{The Department of Neurological Surgery at the University of Wisconsin-Madison}

Since 2006, 219 international trainees from 18 countries and five continents were admitted to the Department of Neurological Surgery at the University of Wisconsin-Madison (Table I). One neurosurgeon received an "International Visiting Surgeon Fellowship" award from the American Association of Neurological Surgeons (AANS) to participate in this training program. Some of these Honorary International Fellows (IFs) were funded from their home country and others were self-funded. The duration of observation for IFs vary from 1 month to 1 year, and it is possible to extend the duration of observership. The main goals for neurosurgical residents and junior neurosurgeons is to learn surgical and cadaveric neuroanatomical approaches, and for senior neurosurgeons to refine and observe advanced surgencies and improve their academic productivity. The core philosophy for the University of Wisconsin fellowship program is that the neurosurgeons who receive training will, in turn, train other neurosurgeons when they return home. Thus, the quality of neurosurgical care will be improved in their regions. A second aspect of the fellowship is to follow the educational model of Avicenna, which is, "to observe."

\section{Application}

The process to become an IF begins with contacting a mentor or meeting a mentor from the University of Wisconsin Neurological Surgery Faculty at an international neurological surgery meeting. Potential IFs then provide application materials, including their curriculum vitae and letters of reference, directly to this mentor. Upon acceptance, the Department of Neurological Surgery and the International Faculty and Staff Services office at the University assists IFs with obtaining necessary approvals and visas.

\section{The First Days of Arrival}

Upon arrival, the commitment of the University is apparent. The new IF is assisted in settling into this new position, and with living in Madison, Wisconsin, USA. Each IF also receives guidance on what is expected from them regarding their position as an IF neurosurgeon, resident, or student.

Table I: Position at Admission to the International Fellows Program at the University of Wisconsin, Madison

\begin{tabular}{lccc}
\hline Position & Number & Percent of Total & Countries of origin \\
\hline Undergraduate & 12 & 5.5 & Brazil, Turkey, USA \\
\hline Medical Student & 82 & 37.4 & Egypt, India, Turkey, USA \\
\hline Medical Doctor & 2 & 0.9 & Iran, Turkey \\
\hline Neurosurgery Resident & 9 & 4.1 & Argentina, Bosnia, Turkey \\
\hline $\begin{array}{l}\text { Neurosurgeon } \\
\text { Total }\end{array}$ & 114 & 52.1 & $\begin{array}{c}\text { Azarbaijan, Brazil, Cameron, Chad, China, Egypt, India, Iran, } \\
\text { Israel, Lithuania, Pakistan, Russia, Turkey, Turkmenistan, Uganda, } \\
\text { Venezuela }\end{array}$ \\
\hline
\end{tabular}




\section{Microsurgery and Neuroanatomy Skill Laboratory}

All IFs are provided with $24 / 7$ access to the Microsurgery and Neuroanatomy Skill Laboratory. This laboratory has two desktop microscopes, three standard operation room (OR) microscopes (one equipped for audio video $3 \mathrm{D}$ recording, including two 3D observation monitors), one endoscope, and other standard neurosurgical OR instruments including craniotomy tools, various drill bits and saws for skull base approaches, and microsurgery instruments. IFs will also have access to cadaveric heads for anatomical study. Cadaveric bodies are also available during annual courses on peripheral nerves and spine surgery (Figure 1).

\section{Clinical and Operation Room Observer}

Neurosurgeons and neurosurgery residents have access to the OR theater, according to their monthly OR schedule. Every OR day, two IFs are committed to attend the OR with their mentor beginning at 7:00 AM until the end of the day's surgeries. During surgery, they are able to directly observe the macroscopic and microscopic steps of surgical procedures in $3 \mathrm{D}$ or $2 \mathrm{D}$ via a live operating microscope video feed (Figure 2). On non-OR days, they review video recordings of these surgeries, and access an extensive library of previously recorded surgeries in both $2 \mathrm{D}$ and $3 \mathrm{D}$ image formats. IFs also learn from other neurosurgeons in diverse subspecialties, neurotologists, vascular neurosurgeons, and other members of intraoperative neurophysiologic monitoring teams. IFs can also attend their mentor's clinic and observe the clinical skills for working with neurosurgical patients.

\section{Research}

Fellows have the opportunity to engage in academic research at the one of the World's leading institutions, on diverse neurosurgical, neuroanatomical, and neuroscience projects. These projects usually arise from the surgical anatomic issues seen in the OR theater. They have the opportunity to publish their studies in peer-reviewed high-impact factor journals. Since the beginning of the IF program, fellows have been authors on 143 journal articles, two books, 21 book chapters and 67 abstracts. IFs also have the opportunity to learn about the management and regulatory aspects of research, including learning about Institutional Review Boards (IRBs), regulations regarding patient privacy (HIPPA), and conflicts of interest.

\section{Microsurgery Training Curriculum}

International neurosurgery residents and neurosurgeons have the opportunity to participate in the "Microsurgery Training Curriculum" to improve their skills in microsurgery and in performing micro-anastomoses. Each participant receives a package of micro-sutures, multiple sizes of anastomosis training model vessels and kits, and appropriate animal tissues for practice. They are taught how to perform end-toend, end-to-side and side-to-side micro-anastomosis. This Training Curriculum and microscope time is free of charge for all participants.

\section{Annually Neurosurgery Courses}

All IFs have the opportunity to participate in the several annual neurosurgery courses sponsored at the University of Wisconsin including, Skull Base, Microsurgery and Microvascular Anastomosis, Peripheral Nerve, and Spinal Anatomy courses. During these courses, IF participants have the opportunity to meet expert neurosurgeons from across the USA who participate as invited faculty, and discuss and share their scientific thoughts in a friendly environment. Participation in these courses is free of charge for IF participants.

\section{Weekly Clinical Meetings, Conferences and Grand Rounds}

All IFs participate in the weekly didactic skull base courses as well as the mortality and morbidity conferences, Grand Rounds, and other announced meetings and courses.

\section{Daily Neurosurgical Intensive Care Unit (ICU) Visits}

All authorized IFs have the opportunity to attend daily neurosurgical intensive care unit (ICU) Rounds with their mentors and observe clinical skills in the neurosurgical critical care patients.

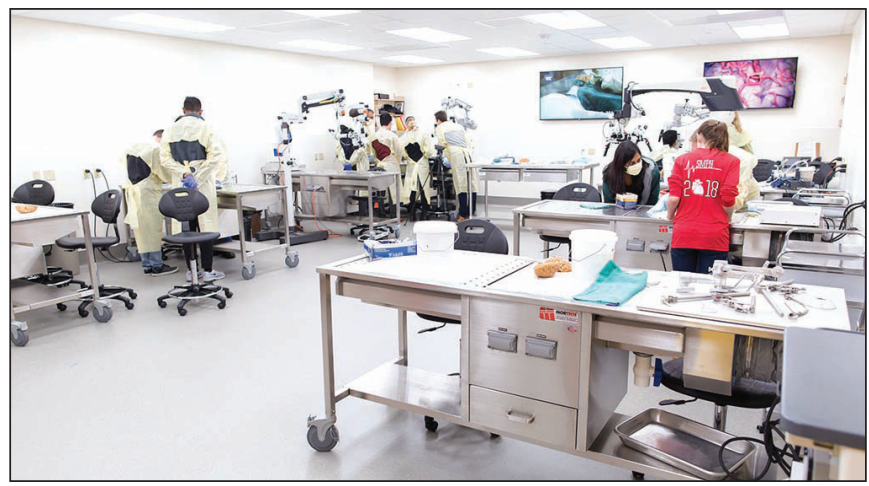

Figure 1: International fellows are provided $24 / 7$ access to the Microsurgery and Neuroanatomy Skill Laboratory.

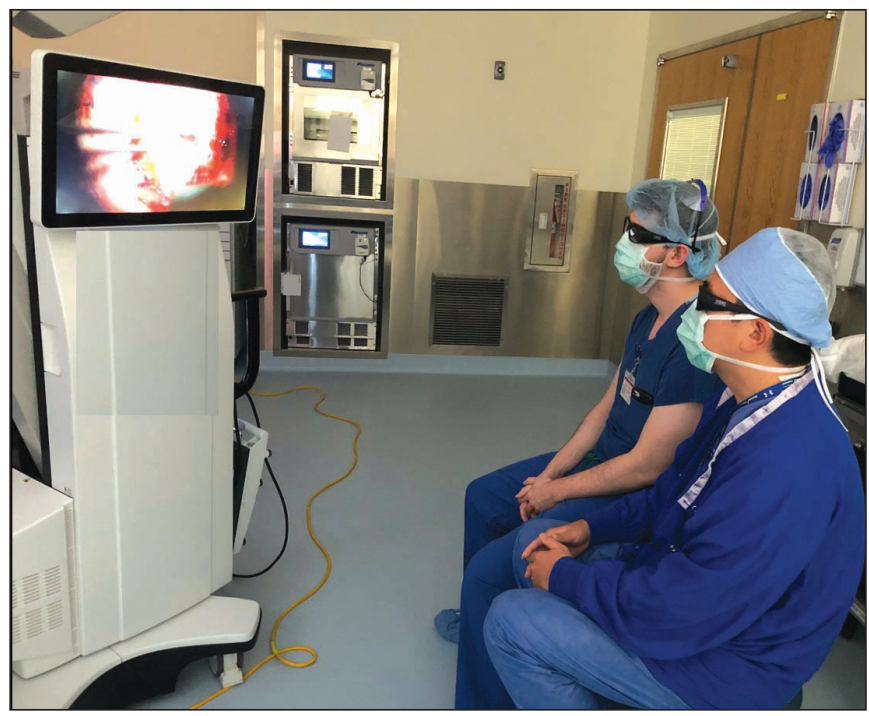

Figure 2: International fellows directly observe macroscopic and microscopic steps of surgical procedures in 2D and 3D via a live operating microscope video feed. 


\section{Diverse, Multicultural, and Friendly Educational Environment}

Participants in the IF program come from all over the world, while the University of Wisconsin faculty, staff, and student body is similarly diverse and multicultural. Fellows will thus meet neurosurgeons, neurosurgery residents, and medical students from around the world (Table I). Daily discussions among IFs often address topics such as, "What is your approach for this pathology?", "Do you have this specific instrument in your hometown?", "Did you perform this procedure in your practice?" and "Do you think you will perform this approach when you go back to your country?". Fellows from non-English language regions will greatly improve their English fluency in such discussions; through participation in journal clubs; through readings in diverse neurosurgical and neuroanatomical areas; and through the group viewing of neurosurgical videos. Fellows with higher levels of training will also commonly share how these cases were treated in their home country to their more junior colleagues and medical students. In this friendly and open environment, fellows will learn from the experience of others that have come from different regions, with different skills, and those having different resources and technical approaches.

\section{Career Advancement}

IFs that have completed the observership program have substantially advanced their careers. Fourteen international neurosurgeons returned to their hometowns with higher academic degree appointments, including two chairman positions, seven professor degrees, two associate professor degrees, two assistant professor degrees, and one consultant neurosurgeon. All of the international neurosurgery residents have reported that their improved knowledge helped their careers. All were also enthusiastic about the future potential to return to the University of Wisconsin to further practice and learn new skills in the Neurosurgical Microsurgery and Neuroanatomy Skill Laboratory. This has been done by one former fellow during their residency, and by two other neurosurgeons that returned multiple times to practice neurosurgical techniques and to perform neuroanatomical research. Medical students that participated in the observership program improved their future prospects for a neurosurgical career, as well as obtained practical and applied neuroanatomical knowledge.

\section{CONCLUSION}

In our rapidly evolving world, neurosurgeons, residents and medical students must be prepared to take a long academic journey to improve their skills in patient care. This is especially true in neurosurgery. When possible, a physical journey can also provide great value to the academic journey, by providing the opportunity to learn new skills and to meet colleagues from diverse programs. The International Fellows program at the University of Wisconsin, Department of Neurosurgery, provides an international venue for neurosurgeons, neurosurgery residents, and medical students to learn advanced skills from experienced faculty, and to learn from each other so that they can bring knowledge back to their home to improve global neurosurgical care. In the 14-plus years of hosting this program, over 200 international scholars have been provided with knowledge and skills to execute this aim, while also being provided an open and friendly educational environment that enables them to maintain world-wide personal relationships that continues to foster excellence for future generations of neurosurgeons world-wide.

One measure of success for the International Fellows Program is that fellows continue to communicate with their mentors and with their prior fellow international colleagues long after their fellowship ends. These professional connections provide a forum to share successes, discuss technique, and to strengthen international cooperation towards advancing neurosurgical patient care.

\section{REFERENCES}

1. Acoustic Neuroma Association of New Jersey [ANA/NJ Newsletter (USA): Vol.Ix, No.2, January 2005]. Available at: https:// www.ananj.org/assets/Jan2005Newsletter.pdf. Accessed October 29, 2019

2. Avicenna. [Wikipedia web site] Available at: https:// en.wikipedia.org/wiki/Avicenna. Accessed October 29, 2019

3. Dempsey RJ: Global neurosurgery: The role of the individual neurosurgeon, the Foundation for International Education in Neurological Surgery, and "service through education" to address worldwide need. Neurosurgical Focus 45(4):E19, 2018

4. Dempsey RJ: Neurosurgery in the developing world: Specialty service and global health. World Neurosurgery 112: 325, 2018

5. Dempsey RJ, Nakaji P: Foundation for international education in neurological surgery (FIENS) global health and neurosurgical volunteerism. Neurosurgery 73(6):1070-1071, 2013

6. Dempsey KE, Qureshi MM, Ondoma SM, Dempsey RJ: Effect of geopolitical forces on neurosurgical training in Sub-Saharan Africa. World Neurosurgery 101:196-202, 2017

7. Dewan MC, Rattani A, Fieggen G, Arraez MA, Servadei F, Boop FA, Johnson WD, Warf BC, Park KB: Global neurosurgery: The current capacity and deficit in the provision of essential neurosurgical care. Executive Summary of the Global Neurosurgery Initiative at the Program in Global Surgery and Social Change. Journal of Neurosurgery 130(4):1055-1064, 2018.

8. Fuller A, Tran T, Muhumuza M, Haglund MM: Building neurosurgical capacity in low- and middle-income countries. Neurological Sci 3:1-6, 2016

9. Meara JG, Leather AJ, Hagander L, Alkire BC, Alonso N, Ameh EA, Bickler SW, Conteh L, Dare AJ, Davies J, Mérisier ED: Global Surgery 2030: Evidence and solutions for achieving health, welfare, and economic development. Lancet 386(9993):569-624, 2015

10. Mukhopadhyay S, Punchak M, Rattani A, Hung YC, Dahm J, Faruque S, Dewan MC, Peeters S, Sachdev S, Park KB: The global neurosurgical workforce: A mixed-methods assessment of density and growth. Journal of Neurosurgery 1(aop):1-7, 2019

11. Park KB, Johnson WD, Dempsey RJ: Global neurosurgery: The unmet need. World Neurosurgery 1(88):32-35, 2016 